\title{
CLARIFICAÇÃO DE SUCO DE MELANCIA ATRAVÉS DE MICROFILTRAÇÃO COM MEMBRANA DE ACETATO DE CELULOSE
}

\author{
M. O. PARAENSE, P. E. AULER, K. C. S. FIGUEIREDO ${ }^{1}$ \\ ${ }^{1}$ Universidade Federal de Minas Gerais, Departamento de Engenharia Química \\ E-mail: katia@deq.ufmg.br
}

RESUMO: O objetivo deste trabalho foi estudar a clarificação do suco de melancia usando membranas comerciais de acetato de celulose 0,45 $\mu \mathrm{m}$. Investigou-se o efeito do fluxo com o tempo, usando escoamento tangencial. As propriedades físico-químicas da alimentação e do

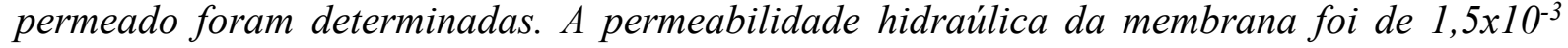
$\mathrm{m} / \mathrm{sPa}$ para a água e de $2,2 \times 10^{-4} \mathrm{~m} / \mathrm{s} P$ a para o suco. O permeado foi submetido a análises de cor, transparência, turbidez, $\mathrm{pH}$, densidade, viscosidade, sólidos solúveis totais e acidez. $\mathrm{O}$ fluxo permeado do suco variou de 61 a $21 \mathrm{~L} / \mathrm{m}^{2} \mathrm{~h}$ durante o experimento. Esta redução esteve associada ao aumento das resistências com o tempo. A resistência da membrana assumiu valores da ordem de $1 \times 10^{6} \mathrm{~m}^{2} / \mathrm{kg}$, enquanto a resistência devido a efeitos de polarização de concentração e a formação da torta assumiram valores uma ordem de grandeza maiores, $10^{7}$ $\mathrm{m}^{2} / \mathrm{kg}$. Não houve resistência irreversível detectável.

PALAVRAS-CHAVE: Processos de separação por membranas; Citrullus lanatus; Polarização de concentração; Torta; Resistência à transferência de massa.

\section{NOMENCLATURA}

A Área da membrana, $\mathrm{m}^{2}$

b Fouling Index

Ja Fluxo permeado mássico de água através da membrana antes da microfiltração do suco de melancia, $\mathrm{Kg} / \mathrm{m}^{2} . \mathrm{s}$

Jp Fluxo permeado mássico do suco de melancia através da membrana, $\mathrm{kg} / \mathrm{m}^{2} . \mathrm{s}$

Mp Massa de permeado, $\mathrm{Kg}$

$\mathrm{Pa}$ Pressão na alimentação, N/m²

Pc Pressão no concentrado, $\mathrm{N} / \mathrm{m}^{2}$ 
Pp Pressão no permeado, $\mathrm{N} / \mathrm{m}^{2}$

$\mathrm{Ri}$ Resistência devida à incrustação, $\mathrm{m}^{2} / \mathrm{kg}$

$\mathrm{Rm}$ Resistência da estrutura da membrana, $\mathrm{m}^{2} / \mathrm{kg}$

t Tempo, h

$\Delta \mathrm{P} \quad$ Variação da pressão, $\mathrm{N} / \mathrm{m}^{2}$

нa $\quad$ Viscosidade da água, N.s $/ \mathrm{m}^{2}$

up Viscosidade do suco de melancia filtrado, N.s $/ \mathrm{m}^{2}$

$\rho \mathrm{p} \quad$ Densidade do permeado, $\mathrm{kg} / \mathrm{m}^{3}$

\section{INTRODUÇÃO}

A melancia (Citrullus lanatus) pertence à família Cucurbitaceae e pode ser considerada terceira fruta mais popular do mundo (Lv et al., 2014). É produzida em áreas livres de longos períodos de gelo e sua utilização é variada, podendo ser na produção de suco, néctar, coquetéis, entre outros (Nabavi-Pelesaraei et al., 2014). No Brasil, em 2013/2014, foram produzidas 2.079.547 toneladas de melancia (Instituto Brasileiro de Geografia e Estatística, 2014), enquanto o mundo produziu 106.400.000 toneladas. A quantidade de melancia produzida no Brasil ocupa o quarto lugar mundial, atrás apenas de China, Peru e Irã, com destaque para a produção nas regiões Nordeste, Norte e Centro-Oeste (Furlaneto e Bertani, 2015).

O suco de melancia representa uma pequena parcela dos sucos vendidos comercialmente, porém contém propriedades que o tornam atrativos para consumo. Ele é fonte de vitamina A e C, de água e de citrulina, aminoácido que participa no sistema de óxido nítrico no corpo humano e apresenta propriedades vasodilatadoras e antioxidantes, e de licopeno- um carotenóide de grande poder antioxidante (Edwards et al., 2003). Estima-se que em $100 \mathrm{~g}$ de bagaço, encontra-se de 3,9 a $7,8 \mathrm{mg}$ de licopeno, o que representa cerca de $60 \%$ a mais do que no tomate. Este apresenta uma alta demanda na indústria farmacêutica, alimentícia e de cosméticos. Ele é um pigmento extremamente sensível ao calor, luz e oxigênio, portanto sua degradação afeta os atributos sensoriais da fruta (Oberoi e Sogi, 2015). O aumento do consumo de citrulina e licopeno a partir do consumo de suco de melancia pode ser uma alternativa promissora, além de agregar valor a uma matéria-prima muito disponível fora do eixo centro-sul do Brasil.

A melancia contém, em massa, de 41 a $60 \%$ de suco (polpa e água), 31 a 59\% de casca e 8 a $23 \%$ de bagaço (polpa), incluindo caroço. Devido à alta umidade da polpa, baixa acidez (pH 5,2-6,7) e alta atividade de água $(0,97-0,99)$, o suco de melancia é suscetível a deterioração microbiana (Oberoi e Sogi, 2015).

$\mathrm{Na}$ indústria, diversos processos são utilizados para preservar a qualidade do suco de melancia e impedir sua deterioração e contaminação por micro-organismos. Tarazona-Diaz e 
Aguayo (2013) investigaram a utilização de acidificação do suco, pasteurização, centrifugação e temperatura de armazenamento a fim de manter as propriedades nutricionais do suco de melancia. Os resultados indicaram que a melhor rota consistiu no uso de produto não centrifugado, armazenado a $4{ }^{\circ} \mathrm{C}$, ao invés de $8{ }^{\circ} \mathrm{C}$. Muito embora a pasteurização tenha diminuído os teores de licopeno, antioxidantes e polifenóis, o uso da centrifugação aumentou ainda mais as perdas, indicando que esta rota não deve ser a preferencial.

Uma das principais aplicações da microfiltração na indústria alimentícia é a clarificação e concentração de sucos de fruta. Desta forma, os sucos podem ser processados em temperatura ambiente, conservando as suas propriedades essenciais para o consumo humano. Entretanto, um dos grandes desafios é compensar o problema do decréscimo do fluxo permeado com o tempo, especialmente do ponto de vista econômico. O estudo das causas desse decréscimo é fundamental para o futuro desenvolvimento de módulos industriais adequados a um processo de clarificação em escala industrial (Chhaya et al., 2008).

\subsection{Microfiltração}

$\mathrm{Na}$ microfiltração, a força motriz para a separação através da membrana é um gradiente de pressão, e as partículas são separadas por exclusão de tamanho (Mulder, 1991). O processo tem potencial para a aplicação na clarificação de sucos, desde que a polarização de concentração, redução reversível do fluxo em função de acúmulo de material na superfície da membrana, e a incrustação, ou fouling, que é a redução irreversível do fluxo devido à diversos mecanismos, como bloqueio de poros, depósito de torta e adsorção, sejam controladas (Baker, 2004).

Dahdouh e colaboradores (2016) investigaram o uso de membranas de microfiltração de poros com tamanho de 0,2 e $2 \mathrm{~mm}$ para a clarificação de suco de laranja. Os autores classificaram o tipo de incrustação presente e relacionaram as mesmas aos tamanhos de partículas presentes no suco, bem como ao tamanho médio de poro das membranas utilizadas.

O uso de pré-tratamentos para evitar a formação de incrustação nas membranas de micro também é uma tendência atual. Domingues e colaboradores (2014) utilizaram a centrifugação, liquefação enzimática e a floculação com quitosana para melhorar o desempenho das membranas face à microfiltração. O pré-tratamento com quitosana foi a rota que apresentou os resultados mais promissores, mas o tipo de incrustação presente foi o de bloqueio dos poros, enquanto os demais caracterizaram-se por depósitos na superfície das membranas.

Considerando a variabilidade dos sucos, Layal e colaboradores (2015) propuseram o uso de um sistema de filtração tipo dead-end em escala laboratorial para prever o tipo de incrustação presente no processo em escala industrial. Os autores utilizaram um planejamento experimental para avaliar os efeitos do tamanho de poros das membranas, velocidade de rotação do impelidor, aceleração centrífuga e tempo de centrifugação. O fluxo permeado médio considerando uma razão de redução da alimentação de 2 foi utilizado como resposta. Os autores concluíram que a ferramenta desenvolvida foi adequada para antecipar o tipo de incrustação do suco de laranja nas membranas cerâmicas avaliadas e propuseram o uso do mesmo procedimento para outros sucos. 
Neste sentido, o objetivo deste trabalho foi avaliar a microfiltração do suco de melancia, a partir do monitoramento do fluxo permeado em função do tempo em um sistema de permeação com escoamento frontal, visando quantificar as resistências ao transporte de massa. As propriedades físico-químicas como cor, acidez e turbidez da alimentação e do permeado foram determinadas para aferir mudanças na qualidade do suco. Não é do nosso conhecimento o uso de dados de microfiltração em membranas poliméricas para a clarificação do suco de melancia.

\section{MATERIAL E MÉTODOS}

\subsection{Preparo da matéria-prima}

A melancia utilizada foi adquirida no comércio local. Foi escolhida de modo que não apresentasse nenhum tipo de defeito na sua casca. Após ser descascada e ter suas sementes removidas, as fatias de melancia foram transferidas para um liquidificador, sem adição de água. Em seguida, a polpa obtida foi peneirada em malha de $38 \mu \mathrm{m}$. A solução de alimentação foi preparada com um teor de $40 \% \mathrm{~m} / \mathrm{m}$ de suco de fruta, com a adição de água destilada. A porcentagem de fruta foi definida com base em Brasil (2003) que estabelece o valor mínimo de $30 \%(\mathrm{~m} / \mathrm{m})$ para néctar. No caso dos sucos de uva e laranja os valores estabelecidos são de $40 \%(\mathrm{~m} / \mathrm{m})$. Assim, foi utilizado este valor como referência e tendência futura para outros tipos de néctar.

\subsection{Testes de permeação}

Para a permeação do suco de melancia, foi utilizada uma membrana de microfiltração de acetato de celulose $0,45 \mu \mathrm{m}$, Millipore. O sistema de permeação é ilustrado na Figura 1. A vazão de alimentação fornecida pela bomba peristáltica XX802 (Millipore), foi de $8 \mathrm{~mL} / \mathrm{min}$. A pressão através da membrana durante o processo de microfiltração foi de $277,7 \mathrm{~N} / \mathrm{m}^{2}$ para a permeação de água destilada, e de $281,1 \mathrm{~N} / \mathrm{m}^{2}$ para a permeação do suco de melancia $40 \%$ $\mathrm{m} / \mathrm{m}$. A área da membrana foi de $0,003 \mathrm{~m}^{2}$ em todos os testes. O volume de alimentação foi de $400 \mathrm{~mL}$.

O fluxo permeado, $\mathrm{J}_{\mathrm{p}}$, foi calculado pela Equação 1:

$$
I_{p}=\frac{M_{p} / \rho_{p}}{A \times t}
$$

onde, A é a área da membrana $\left[\mathrm{m}^{2}\right], \mathrm{M}_{\mathrm{p}}$ é a massa de permeado $[\mathrm{kg}], \rho_{\mathrm{p}}$ é a densidade do permeado $\left[\mathrm{kg} / \mathrm{m}^{3}\right]$ e t é o tempo $[\mathrm{h}]$. 


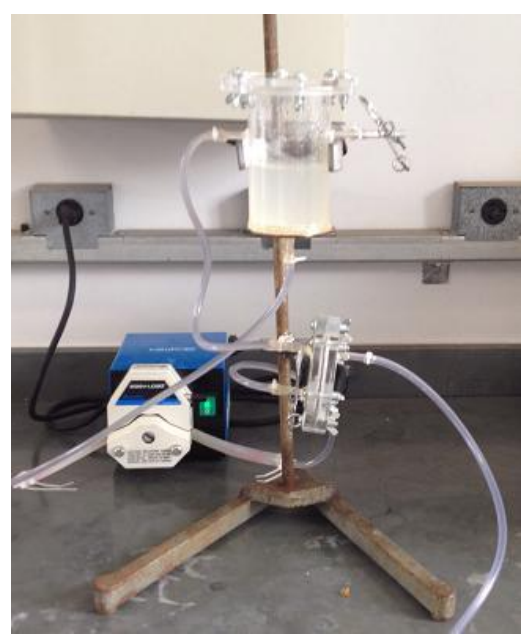

Figura 1- Sistema de permeação por membrana de microfiltração.

O fluxo permeado foi utilizado no cálculo da resistência da membrana e da resistência devido à incrustação, baseando-se no modelo das resistências descrito por Baker (2004), de acordo com a Equação 2:

$$
R m=\frac{\Delta P}{\mu a \times J a}
$$

onde $\mu$ a é a viscosidade da água $\left[\mathrm{N} . \mathrm{s} / \mathrm{m}^{2}\right]$ e Ja é o fluxo permeado de água através da membrana antes da microfiltração do suco de melancia $\left[\mathrm{kg} / \mathrm{m}^{2} . \mathrm{s}\right]$. Após processar o suco de melancia, a resistência devida à incrustação pode ser calculada de acordo com a Equação 3:

$$
R i=\frac{\Delta P}{\mu p \times J p}-R m
$$

onde, $\mu$ p é a viscosidade do suco de melancia filtrado [N.s $\left./ \mathrm{m}^{2}\right]$ e Jp é o fluxo permeado do suco de melancia através da membrana $\left[\mathrm{kg} / \mathrm{m}^{2} . \mathrm{s}\right]$.

A solução foi alimentada no tanque e separada por meio da filtração tangencial, tendo a sua massa anotada no intervalo de 3 minutos até completar 42 minutos. Os testes de permeação foram feitos em triplicata.

Com a amostra já permeada, as análises de densidade (011/IV), viscosidade, acidez (253/IV), turbidez (206/IV), pH (201/IV), cor (A420), transparência (T660) e sólidos solúveis totais (203/IV) foram realizadas para todas as soluções permeadas, para a solução de alimentação e para o suco de melancia antes de sofrer um pré-tratamento. Cabe ressaltar, que as análises físico-químicas foram executadas de acordo com normas e procedimentos descritos pelo Instituto Adolfo Lutz (2008). Todas as análises foram realizadas em triplicata para o permeado e para a alimentação, na pressão ambiente e temperatura de $30{ }^{\circ} \mathrm{C}$, em até 24 horas após o experimento. 


\section{RESULTADOS E DISCUSSÃO}

\subsection{Fluxo do permeado}

A massa do permeado foi medida durante a microfiltração do suco. Estes dados foram utilizados para calcular o fluxo permeado no tempo, conforme Figura 2.

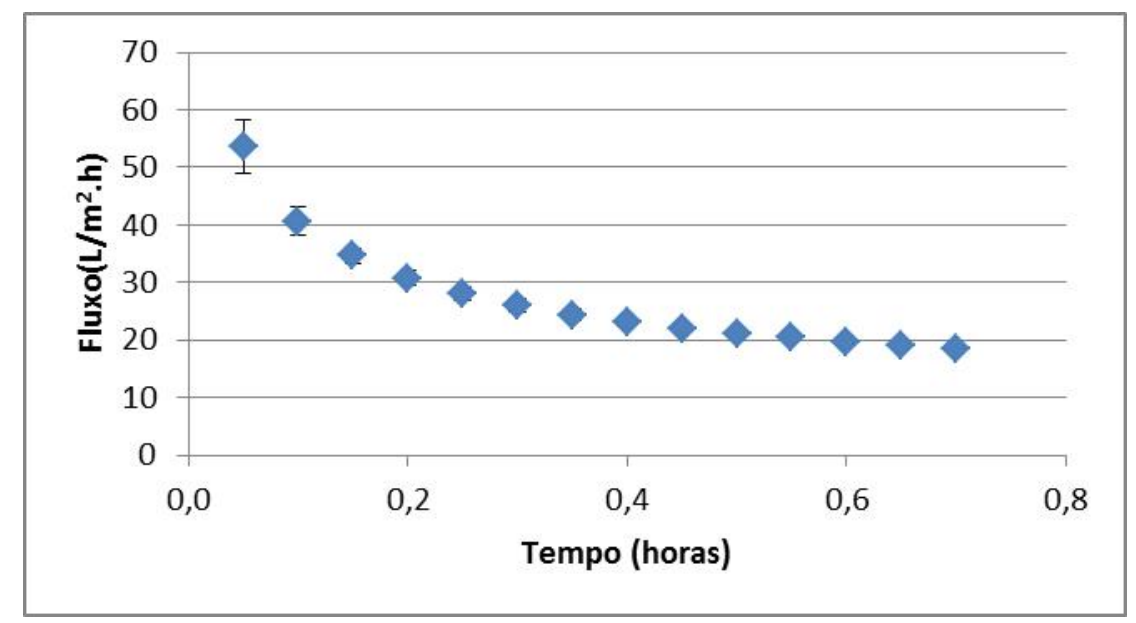

Figura 2 - Fluxo permeado no tempo durante a microfiltração do suco de melancia.

O fluxo permeado diminuiu em 48,6\%. No início, o fluxo foi de $(61 \pm 5) \mathrm{L} / \mathrm{m}^{2} \mathrm{~h}$, após 42 minutos seu valor atingiu $(21,5 \pm 0,7) \mathrm{L} / \mathrm{m}^{2} \mathrm{~h}$. Isto ocorreu devido à existência de resistências à transferência de massa através da membrana, como a resistência da membrana e a resistência causada pelos efeitos de polarização de concentração, formação de torta e incrustação (Habert et al., 2006). Logo, as resistências à transferência de massa foram menores no início da microfiltração do suco de melancia e aumentaram com o tempo, de acordo com o esperado.

A queda inicial do fluxo está associada à resistência polarização de concentração, que se fosse o único efeito responsável pelo decréscimo do fluxo, levaria este a uma estabilização. Porém, como o sistema não atinge um fluxo constante, observam-se efeitos de incrustações (Chhaya et al., 2008). Na prática, foi possível observar a formação de uma torta sobre a superfície da membrana após a microfiltração.

\subsection{Resistência à Transferência De Massa}

Antes da microfiltração, a membrana foi permeada com água destilada. Após 2 minutos, o sistema atingiu o regime permanente e apresentou um valor do fluxo de água permeada constante no tempo, $(0,07 \pm 0,01) \mathrm{kg} / \mathrm{m}^{2} \mathrm{~s}$. Com os dados do fluxo em função do tempo, a resistência da membrana foi calculada. O valor obtido foi de $(5,7 \pm 0,9) \times 10^{6} \mathrm{~m}^{2} / \mathrm{kg}$.

No processamento do suco de melancia através da microfiltração, os valores do fluxo em função do tempo foram utilizados para o cálculo da resistência devido ao fouling, representada na Figura 3. Esta resistência aumentou com o tempo, e a taxa de aumento foi 
maior nos momentos iniciais devido à formação da torta. Seus valores iniciais foram de $(9 \pm$ 2) $\times 10^{6} \mathrm{~m}^{2} / \mathrm{kg}$ e após o final do procedimento seu valor foi de $(3,6 \pm 0,1) \times 10^{7} \mathrm{~m}^{2} / \mathrm{kg}$. Portanto, a resistência total ao transporte aumentou com o tempo, e é uma ordem de grandeza maior que a resistência da membrana $\left(10^{7}\right.$ contra $\left.10^{6} \mathrm{~m}^{2} / \mathrm{kg}\right)$.

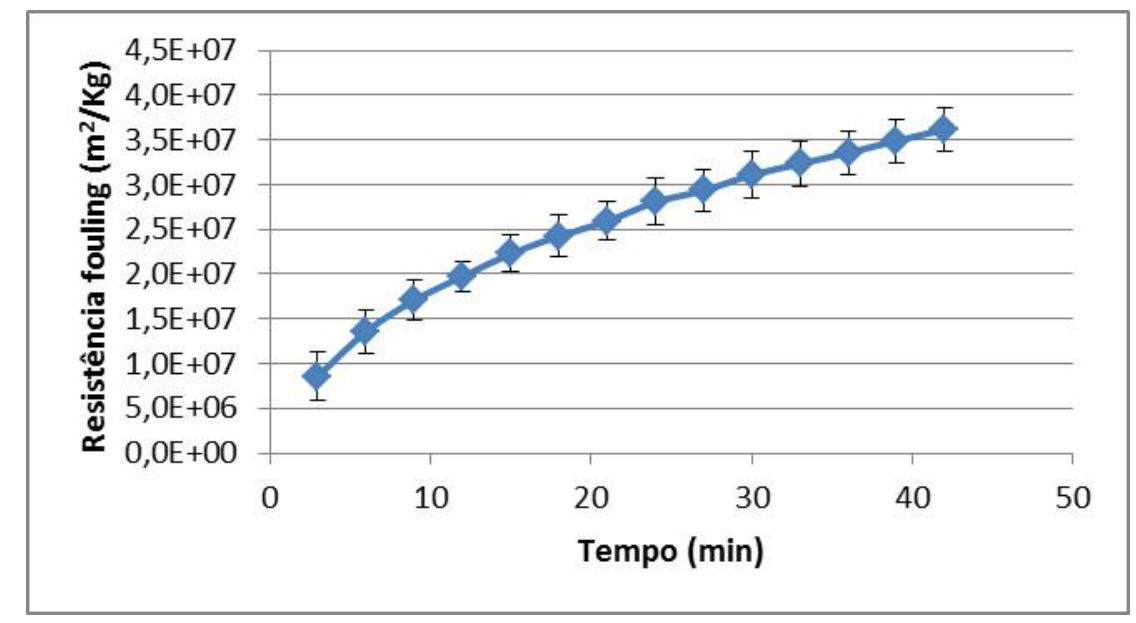

Figura 3 - Resistência em função do tempo, durante a microfiltração do suco de melancia.

Após a utilização das membranas, estas foram lavadas com água, de forma a retirar a torta da superfície. Assim, as membranas foram novamente permeadas com água destilada com a finalidade de calcular a resistência irreversível ao processo. $\mathrm{O}$ valor do fluxo de água permeada foi de $(0,09 \pm 0,01) \mathrm{kg} / \mathrm{m}^{2} \mathrm{~s}$, indicando que o fluxo inicial foi recuperado e que não houve resistência irreversível no processo.

\subsection{Fouling index}

O efeito de fouling na membrana pode ser modelado no tempo através da variação do fluxo permeado do suco de melancia em relação ao fluxo de água, como representado na Equação 4 (Mirsaeedghazi et al., 2010):

$$
b=\log _{t}\left(\frac{J a}{J p}\right)
$$

onde, Jp é o fluxo permeado do suco de melancia através da membrana $\left[\mathrm{kg} / \mathrm{m}^{2} . \mathrm{s}\right]$, Ja é o fluxo permeado de água através da membrana antes da microfiltração do suco de melancia $\left[\mathrm{kg} / \mathrm{m}^{2} . \mathrm{s}\right]$, t é o tempo [min] e b é o fouling index.

No início da microfiltração, a formação da torta é a principal respónsavel pela resistência ao processo de clarificação e pela queda no fluxo permeado, traduzido no elevado valor do fouling index. Após a completa formação da torta, o principal efeito de resistência passa a ser o de polarização de concentração e possíveis incrustações que são efeitos secundários e de menor impacto na redução do fluxo permeado. Assim, nos estágios finais do processamento do suco de melancia, o fouling index se estabiliza com o tempo, em valores próximos a 0,65, como ilustrado na Figura 4. 


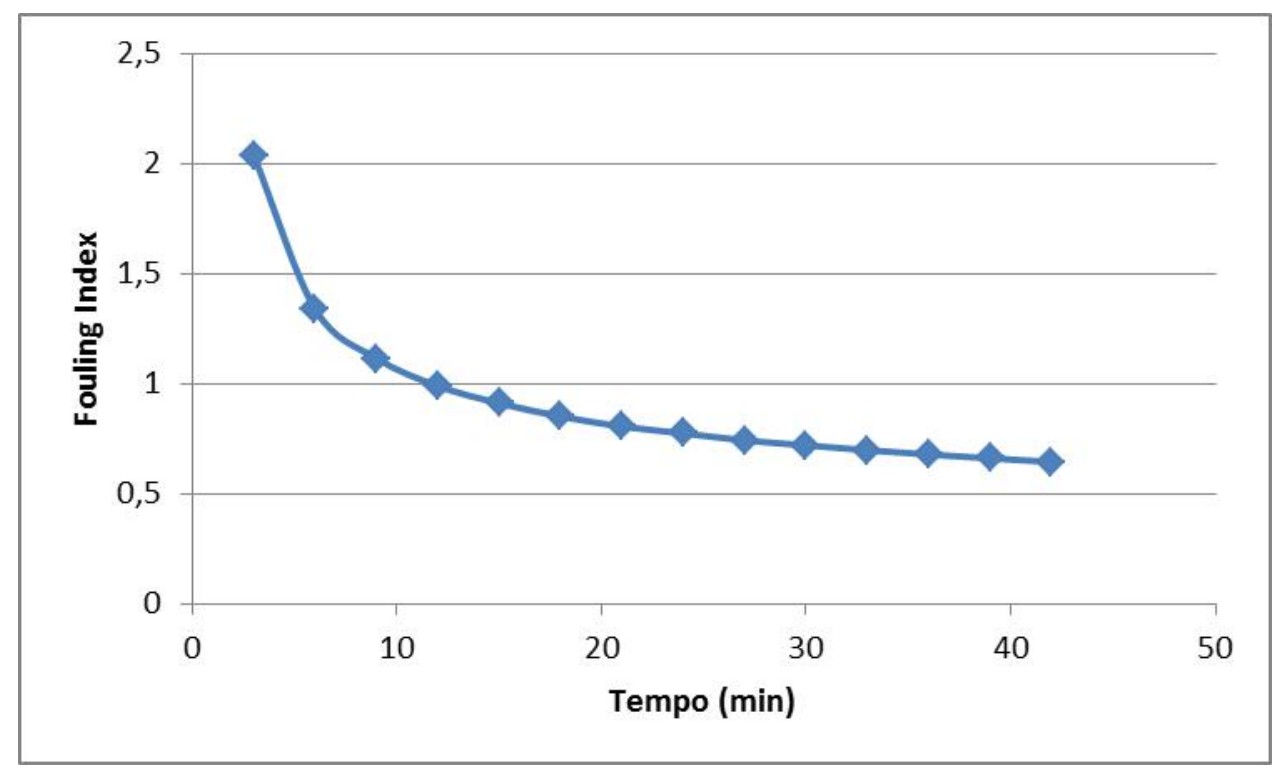

Figura 4 - Comportamento do fouling index no tempo, durante a microfiltração do suco de melancia.

\subsection{Propriedades da alimentação e do permeado}

Os valores obtidos para as propriedades físico-químicas da alimentação e do permeado são apresentados na Tabela 1. Todas as medidas apresentaram desvios menores do que $10 \%$.

A propriedade turbidez sofreu um decréscimo de 99,2\%. Isto indica que a microfiltração foi capaz de remover espécies que causam a turbidez, as partículas de sólidos solúveis com tamanho acima de $0,45 \mu \mathrm{m}$, que representam apenas cerca de $0,4{ }^{\circ} \mathrm{Brix}(9 \%)$ dos sólidos solúveis totais presentes na alimentação. Estes sólidos são compostos por polissacarídeos como lignina, celulose e pectina que ficam retidos na torta (Barros et al., 2003; Yu et al., 1986). Mais além, os sólidos solúveis totais (TSS) sofreram redução de 9\%. Este resultado é uma consequência da remoção dos sólidos solúveis que causam parte da turbidez do suco.

A intensidade de cor do suco alimentado ao sistema mostrou decréscimo de $93 \%$ e a transparência apresentou um aumento de $94 \%$, após o processamento do suco. Este resultado demonstra que a microfiltração é capaz de clarificar o suco de melancia, devido à remoção de partículas coloidais suspensas presentes na alimentação (Chhaya et al., 2008).

$\mathrm{O}$ pH não sofreu alteração significativa em seu valor comparando o suco de melancia fresco e o permeado. Este resultado era esperado, visto que uma membrana de microfiltração não é capaz de remover os íons $\mathrm{H}^{+}$de uma solução (Baker, 2004). O pequeno aumento no $\mathrm{pH}$ é respaldado pela pequena queda na acidez, e causado pela retenção de uma fração de acidez em forma de moléculas de ácido (Chhaya et al., 2008). Adicionalmente, a acidez medida em gramas de ácido cítrico indicou queda de $14 \%$. A diminuição da acidez tem associação com o fato de a concentração do ácido ascórbico diminuir com o tempo devido a oxidação, como reportado por Chhaya et al. (2008), sendo este o principal ácido presente no suco de melancia. 
Tabela 1 - Propriedades físico-químicas da alimentação e do permeado.

\begin{tabular}{ccc}
\hline Propriedade & Alimentação & Permeado \\
\hline Turbidez (NTU) & 410,2 & 3,1 \\
Cor & 1,4 & 0,1 \\
Transparência & 5,9 & 98,4 \\
$\mathbf{p H}$ & 5,6 & 5,8 \\
Densidade $(\mathbf{g} / \mathbf{m L})$ & 1,01 & 1,01 \\
\hline Viscosidade cinemática $\left(\mathbf{m m}^{\mathbf{2}} / \mathbf{s}\right)$ & 1,1 & 0,8 \\
\hline Acidez $(\mathbf{g}$ ácido cítrico $/ \mathbf{1 0 0} \mathbf{m l})$ & 0,07 & 0,06 \\
TSS $\left({ }^{\circ}\right.$ Brix) & 4,6 & 4,2 \\
\hline
\end{tabular}

A viscosidade cinemática diminuiu em $27 \%$. Isto ocorreu devido à remoção de todos os sólidos suspensos durante o processamento do suco de melancia, os quais são responsáveis pela viscosidade maior da alimentação (Sogi, 2003). A viscosidade final do permeado é próxima à viscosidade do solvente, $0,801 \mathrm{~mm}^{2} / \mathrm{s}$ para a água destilada a $30{ }^{\circ} \mathrm{C}$.

A microfiltração não alterou a densidade do suco de melancia devido ao valor desta densidade ser próximo do valor da densidade do solvente, $0,99 \mathrm{~g} / \mathrm{mL}$, para a água destilada à $30{ }^{\circ} \mathrm{C}$ (Perry e Green, 1992).

\section{CONCLUSÃO}

Os resultados obtidos indicam que o suco de melancia foi clarificado com sucesso, utilizando a microfiltração através de membranas de acetato de celulose $0,45 \mu \mathrm{m}$. A clarificação está evidenciada pela alteração na turbidez, cor e transparência: a turbidez apresentou um decréscimo de $99 \%$, a intensidade de cor sofreu redução de $93 \%$, a transparência aumentou em 94\%. As demais propriedades físico-químicas não apresentaram alterações significativas. Vale ressaltar que não foi detectada queda irreversível no fluxo permeado da membrana, indicando o potencial do sistema para aplicações em escala industrial. 


\section{AGRADECIMENTOS}

Agradecemos a todos os colaboradores deste artigo, a FAPEMIG, ao Capes e ao CNPQ pelo financiamento à pesquisa.

\section{REFERÊNCIAS}

BAKER, R. W. Membrane Technology and Applications. 2. ed. Chichester, UK: John Wiley \& Sons Ltd, 2004.

BARROS, S. T. D.; DE ANDRADE, C. M. G.; MENDES, E. S.; PERES. L. Study of fouling mechanism in pineapple juice clarification by ultrafiltration. J. Membr. Sci., v. 215, p. 213-224, 2003.

BRASIL. Ministério da Agricultura, Pecuária e Abastecimento. Instrução Normativa $n^{\circ} 42$, de 11 de Setembro de 2013. Art. 87, parágrafo único do inciso II, da Constituição, do Decreto $\mathrm{n}^{\mathrm{o}}$ 6.871, de 4 de junho de 2009. Diário Oficial da União da República Federativa do Brasil, Brasília, DF, 12 set. 2013.

CHHAYA, Ch.; RAI, P.; MAJUMDAR, G. C.; DASGUPTA, S.; DE, S. Clarification of watermelon (CITRULLUS LANATUS) juice by microfiltration. J. Food Eng., v. 31, p. 768-782, 2008.

DAHDOUH, L.; DELALONDE, M.; RICCI, J.; SERVENT, H.; DORNIER, M.; WISNIEWSKI, C. Size-cartography of orange juices foulant particles: contribution to a better control of fouling during microfiltration. J. Memb. Sci., v. 509, p. 164-172, 2016.

DOMINGUES, R. C. C.; RAMOS, A. A.; CARDOSO, V. L.; REIS, M.H.M. Microfiltration of passion fruit juice using hollow fibre membranes and evaluation of fouling mechanisms. J. Food Eng., v. 121, p. 73-79, 2014.

EDWARDS, A.J.; VINYARD B.T.; WILEY, E.R.; BROWN E.D.; COLLINS, J.K.; PERKINSVEAZIE, $P$. Consumption of watermelon juice increases plasma concentrations of lycopene and $\beta$-carotene in humans. J Nutr , v. 133, p. 1043-1050, 2003.

FURLANETO, F. P. B., BERTANI, R. M. A., Melancia - Do Brasil para o mundo. Revista Campo \& Negócios - Hortifrúti, v. 8, n. 4, pp. 13-15, 2015.

HABERT, S. C.; BORGES, C. P.; NOBREGA, R. Processos de Separação por Membranas. 1 ed. Rio de Janeiro: COPPE/UFRJ, E-papers, 2006.

INSTITUTO ADOLFO LUTZ .Métodos físico-químicos para análise de alimentos. 1. ed. digital. São Paulo: Instituto Adolfo Lutz, 2008.

INSTITUTO BRASILEIRO DE GEOGRAFIA E ESTATÍSTICA. Produção Agrícola Municipal: culturas temporárias e permanentes. Rio de Janeiro, v. 41, 2014. Disponível em: <http://biblioteca.ibge.gov.br/visualizacao/periodicos/66/pam_2014_v41_br.pdf $>$. Acesso em: 03 maio. 2017.

LAYAL, D.; CHRISTELLE, W.; JULIEN, R.; ANDRÉ, K. G.; MANUEL, D.; MICHELE, D. Development of an original lab-scale filtration strategy for the prediction of microfiltration performance: application to orange juice clarification. Sep. Pur. Tech., v. 156, p. 42-50, 2015.

LV, P.; LI, N.; LIU, H.;GU, H.; ZHAO, W.; Changes in carotenoid profiles and in the 
expression pattern of the genes in carotenoid metabolisms during fruit development and ripening in four watermelon cultivars. Food Chemistry, v. 174, p. 52-59, 2014.

MIRSAEEDGHAZI, H.; EMAM-DJOMEH, Z.; MOUSAVI, S. M.; AROUJALIAN, A.; NAVIDBAKHSH, M. Clarification of pomegranate juice by microfiltration with PVDF membranes. Desalination, v. 264, p. 243-248, 2010.

MULDER, M. Basic Principles of Membrane Technology. 1 ed. Netherands: Kluver Academic Publishers. 1991

NABAVI-PELESARAEI A.; ABDI R.; RAFIEE S.; BAGHERI I.; Determination of efficient and inefficient units for watermelon production-a case study: Guilan province of Iran, Journal of the Saudi Society of Agricultural Sciences,2014.

OBEROI, D. P. S.; SOGI, D. S; Prediction of lycopene degradation during dehydration of watermelon pomace (cv Sugar Baby). Journal of the Saudi Society of Agricultural Sciences, 2015. In Press.

PERRY, R. H.; GREEN, D .W. Perry's Chemical Engineers' Handbook. Mc Graw-Hill, New York, 50th edition edition, 1992.

SOGI, D.S. Effect of concentration and temperature on the viscosity of watermelon juice. $\mathbf{J}$. Food Sci. Technol, v. 40, n. 5, p. 509-511, 2003.

TARAZONA-DIAZ, M. P.; AGUAYO, E. Influence of acidification, pasteurization, centrifugation and storage time and temperature on watermelon juice quality. J Sci Food Agric, v. 93, p. 3863-3869, 2013

YU, Z. R.; CHIANG, B. H.; HWANG, L. S. Retention of passion fruit juice compounds by ultrafiltration. J. Food Sci., v. 51, p. 841-844, 1986.

\title{
CLARIFICATION OF WATERMELON JUICE BY MICROFILTRATION WITH CELLULOSE ACETATE MEMBRANES
}

\begin{abstract}
The objective of this paper was to study the process of clarification of watermelon juice utilizing commercial membranes made of cellulose acetate 0,45 $\mu \mathrm{m}$. The permeate flux as function of time was evaluated in a tangent flow mode. The physicochemical properties of feed and permeate were addressed. The hydraulic permeability of the membrane was $1.5 \times 10^{-3} \mathrm{~m} / \mathrm{sPa}$ considering water as the fluid and $2.2 \times 10^{-4} \mathrm{~m} / \mathrm{sPa}$ considering the juice. The properties analyzed in the permeate juice were color, transparency, turbidity, $\mathrm{pH}$, density, viscosity, total soluble solids and acidity. The permeate flux of the juice assumed values from 61 to $21 \mathrm{~L}^{2} \mathrm{~m}^{2} \mathrm{~h}$ during the experiment. This reduction was linked to the increase of the resistances with time. The membrane resistance assumed values of $1 \times 10^{6} \mathrm{~m}^{2} / \mathrm{kg}$, while the resistance associated with concentration polarization and cake formation assumed values one order of magnitude higher, $10^{7} \mathrm{~m}^{2} / \mathrm{kg}$. It was not possible to detect irreversible resistance.
\end{abstract}

KEYWORDS: Membrane separation processes; Citrullus lanatus; Concentration polarization; Cake; Mass transfer resistances. 